\section{Forces in cell biology}

\section{Mechanobiology - the study of how physical forces control the behaviour of cells and tissues - is a rapidly growing field. In this issue, we launch a Series of specially commissioned Review articles that discuss exciting recent developments in this area.}

Cell behaviour is guided not only by chemical signals, but by the mechanical properties of the cells and their environment. Cells are able to sense and transduce external mechanical inputs into biochemical and electrical signals that influence processes such as cell proliferation, adhesion, migration and fate. Such mechanotransduction is important in development and homeostasis and, importantly, affects the progression of diseases including muscular dystrophies, cardiomyopathies, fibrosis and cancer. Although our understanding of the specific mechanisms of force sensing and transduction is still limited to the more tractable biological systems, technological advances have contributed to the rapid growth of this multidisciplinary field. Mechanobiology combines in vitro, cell-based work with ex vivo and in vivo experiments at the tissue and organismal level, and brings together classic biology, engineering and physics, to address fundamentally cell biological questions about how mechanics affect cellular processes. As such it has emerged as one of the most exciting areas within the scope of Nature Cell Biology in the last decade.

In light of its significant growth and the high interest of this field to our broad cell biology audience, we are delighted to launch a Series of specially commissioned Review articles discussing recent advances, with a Review by Carl-Philipp Heisenberg, accompanied by Research Highlights on recent mechanobiology studies published elsewhere. The Series can be found on a dedicated page of our website (http://go.nature.com/2pFbjPS) where readers can also access an online library comprised of related articles published in Nature Cell Biology and other Nature journals.

In this issue, Heisenberg and co-authors review our current understanding of how mechanonsensing and mechanotransduction occur in the context of tissue organization during embryogenic development. For many developmental processes, a mechanical understanding is still lacking, but some morphogenetic processes have been studied in more detail. The authors provide an overview of concepts that have emerged from studying such systems, including germband extension and the shaping of imaginal discs in Drosophila as well as Xenopus gastrulation and zebrafish epiboly. This Review also serves as an excellent introduction to how cells perceive and transduce force.

The mechanobiology field has been driven forward largely thanks to the development of sophisticated methods to probe and quantify the response to forces by cells and tissues, ranging from microscopy-based tools to molecular force sensors. Simultaneously, the use of microfabricated devices has made possible the manipulation of cellular constraints. Next month, Pere Roca-Cusachs and Xavier Trepat will discuss the methods used to measure the forces generated by cells, and their applicability in the laboratory.

The site of a cell's adhesion to the underlying matrix or to other cells is central for coupling the extracellular matrix or neighbouring cells to cell-intrinsic mechanosensing structures, to generate mechanical feedback and/or translate forces into biochemical signals. Future Reviews in the Series will discuss such events and how they relate to cell migration, cell shape and growth, and nuclear events such as chromatin organization and transcription, in development, homeostasis and disease.

Much remains to be discovered in this stimulating field at the crossroads of biology, biophysics, bioengineering, and we look forward to Nature Cell Biology continuing to be a key outlet for this flourishing area of research. We thank our authors and referees for their contributions, and hope that this Series serves as both information and inspiration for our readers.

\section{Championing authorship attribution}

Nature Cell Biology is among the Springer Nature journals taking part in a recently launched trial that mandates the provision of ORCID identifiers for the corresponding authors of our papers.

Receiving credit for one's publications is central in scientific research and publishing. However, researchers may share the same name, or publish under different names over the course of their careers. In 2010, the Nature Publishing Group and Springer were among the founding members of ORCID (Open Researcher and Contributor ID) - an open, not-for-profit organization that provides individuals with a unique, persistent ORCID identifier, an ORCID iD that can be consistently associated with their name (in its various iterations), affiliations and their research work. Obtaining an ORCID iD is free and simple to do through the ORCID registration page (https://orcid. org/register) and 128,349 of our authors and referees across Nature Research have done so in the last seven years.

We have now taken an additional step in our efforts to ensure that researchers are recognized and receive credit for their work: Nature Cell Biology is one of the 14 Nature journals, and 46 Springer Nature journals that are participating in a trial mandating that the corresponding authors of papers accepted for publication as of 27 April 2017 provide their ORCID iDs. Authors are being prompted to provide their ORCID iD during the manuscript submission process, and are also able to do so at any point prior to the manuscript's acceptance for publication. The trial will last for six months, at the end of which we will evaluate the feedback we receive. We welcome your comments on this initiative at ncb@nature.com. 